\title{
Accurate Permeability Prediction in Tight Gas Rocks via Lattice Boltzmann Simulations with an Improved Boundary Condition
}

\author{
Dian Fan \\ Department of Chemical Engineering, University College London, London WC1E 7JE, U.K.
}

\begin{abstract}
Accurately predicting gas transport in rocks is required for enhancing the accuracy of field production models. The mesoscale lattice Boltzmann (LB) method can be implemented to predict gas permeability in porous rocks. However, the published LB results for the Klinkenberg effect are often inconsistent with the widely used Beskok-Karniadakis-Civan's (BKC) correlation. The culprit of the unphysical effect has been identified in the typically implemented boundary conditions (BCs). An improved BC is proposed herein to reliably predict gas permeability. Non-equilibrium molecular dynamics simulations are conducted to benchmark the proposed approach. The results show that the presented LB predictions for the Klinkenberg effect are quantitatively consistent with experimental data and the BKC correlation, indicating that the unphysical effects have been minimized. More importantly, a numerical consistency is achieved for describing the Klinkenberg effect at molecular through macroscopic scales. These observations are relevant for improving our ability to predict gas production from tight formations.
\end{abstract}

$\uparrow$ Corresponding author: d.fan@ucl.ac.uk

† Corresponding author: a.striolo@ucl.ac.uk 


\section{Introduction}

The significance of gas permeability estimation cannot be overstated for hydrocarbon gas recovery (Striolo and Cole 2017). The accuracy of large-scale production models for the tight formation including shale formation depends on assumptions regarding the permeability of the rock mass (Liu et al. 2019, Liu et al. 2019, Rani et al. 2019, Xu et al. 2019). However, predicting gas permeability through these rocks remains challenging because of the need to balance numeral accuracy and computational cost (Apostolopoulou et al. 2019, Bonnaud et al. 2012, Bui et al. 2017, Fan et al. 2002, Phan et al. 2016, Qomi et al. 2014). Among alternative approaches, lattice Boltzmann (LB) simulations are effective in studying fluid transport at intermediate length and time scales (i.e., the mesoscale) at a modest computational cost (Fathi et al. 2012, Krüger et al. 2017, Landry et al. 2016, Moghaddam and Jamiolahmady 2017, Ren et al. 2015, Succi and Succi 2018, Wang et al. 2016, Zhao et al. 2016, Zhao et al. 2016). In this work, we consider the LB approach, as described in what follows.

The lab-measured rock permeability by gas flood is defined as the apparent permeability of the rock. When gas flows through a porous medium, a slip velocity could occur on pore surfaces and the resultant apparent gas permeability exceeds the rock intrinsic permeability, i.e., the permeability that reflects the topology of the pore network regardless to the type of the fluid flowing through. The apparent permeability is dependent on the Knudsen number $(\mathrm{Kn})$, which is defined as the ratio of the mean free path of a gas molecule $(\lambda)$ to the characteristic length available to the gas within the transport system $(l)$ (Maurer 
et al. 2003). The $K n$-dependent permeability is known as 'Klinkenberg effect' (Klinkenberg 1941). The Klinkenberg effect becomes pronounced as $K n$ increases, which could occur in shales and tight sandstones (Ziarani and Aguilera 2012). When $K n>0.001$, gas frequently collides with the solid walls and solid-gas interactions become significant for determining the apparent permeability (Darabi et al. 2012, Fan and Ettehadtavakkol 2017).

In the past decade, the LB method has been applied to simulate fluid flows at finite $K n$ values (Toschi and Succi 2005). It has been recognized that the boundary conditions (BCs) are essential for ensuring that LB simulations yield realistic results at such conditions (Succi 2016). Some attempts on BCs have been made for the finite-Kn flow in the past decades, including the specular reflection BC (Chapman et al. 1990), the diffusive BC (Ansumali and Karlin 2002, Niu et al. 2007), the combined BC obtained by full diffusive and specular reflection (Tang et al. 2005b), and that obtained by bounce-back and specular reflection (Succi et al. 2002). Perhaps among other BCs, the bounce-back (BB) and specular reflection (SR) is of greatest interest for predicting gas permeability in shale rocks. This is because the $\mathrm{BB}+\mathrm{SR} \mathrm{BC}$ can apply the non-unitary values of the tangential momentum accommodation coefficient (TMAC), which reflects realistic gas-wall interactions for gases typically used in shale core-flooding experiments (Maurer et al. 2003, Wu et al. 2017, Yamaguchi et al. 2017). The BB BC and the SR BC are intended to simulate no-slip and infinite gas slip phenomena, respectively. 
Although some studies (Guo et al. 2008, Tang et al. 2005a) attempted to investigate the accuracy on the application of these BCs for finite- $K n$ flow, direct comparison against MD simulation results and experimental permeability data, especially for complex porous media, in general lacked. Therefore, the thorough validation of the classical slip BCs for finite- $K n$ flow has been somewhat incomplete. In fact, recent applications to shale rock samples (Chen et al. 2015, Li et al. 2018, Zhao and Wang 2019) that implemented the combined BB+SR BC yield overestimated Klinkenberg effects compared to the BeskokKarniadakis-Civan's (BKC) correlation (Beskok and Karniadakis 1999, Civan 2010). The BKC's correlation combines the permeability correction correlation from Ref. (Beskok and Karniadakis 1999) and the rarefaction coefficient from Ref. (Civan 2010), and is one of the commonly applied correlations for the permeability correction factor in tight rocks. To date, few studies probe into the reason for such overestimation. It was mentioned that the pore network complexity may account for the observed difference, but further validation is missing (Chen et al. 2015, Zhao and Wang 2019).

One major component of the $\mathrm{BB}+\mathrm{SR} \mathrm{BC}$ is the $\mathrm{BB}$ part. Previous studies found that the BB BC does not always achieve zero velocity at the wall, suggesting that its misuse could lead to predicting unphysical slip (Prestininzi et al. 2016, Verhaeghe et al. 2009). The unphysical slip introduced by implementing the $\mathrm{BB} B \mathrm{BC}$ is found to depend on fluid viscosity (Noble et al. 1995). One approach to overcome the BB BC limitations is the consistent hydrodynamic $\mathrm{BC}$ (HBC), which can minimize the unphysical slip at the corner of the octagonal boundaries (Gallivan et al. 1997). Another approach recently proposed is 
to control the velocity sets parallel to the solid surface (Meng et al. 2018), which is based on the theoretical studies that suggest a no-slip condition could only be achieved when local momentum density at the wall is zero (He et al. 1997). We speculate that the reason for the overestimation of the Klinkenberg effect when implementing the combined BB+SR $\mathrm{BC}$ can be due to the unphysical slip from the BB component. For clarity, we thereafter name the standard $\mathrm{BB}(\mathrm{SBB})$ and the classical combined $\mathrm{BB}+\mathrm{SR} \mathrm{BC}$ as the classical $\mathrm{BC}$ for no-slip flow and slip flow in this work.

This paper addresses two fundamental questions by simulating gas flows through micro/nano-porous media via non-equilibrium MD simulations and LB simulation sequentially, where MD simulations are used to benchmark the LB results to identify the appropriate BCs: (1) whether the LB method is applicable to describe gas flows through micro-/nanoporous media, and (2) whether the physical phenomena responsible for the Klinkenberg effect are consistently described at microscopic, mesoscopic, and macroscopic scales. An improved $\mathrm{BC}$ is introduced for $\mathrm{LB}$ calculations to reproduce non-equilibrium $\mathrm{MD}$ simulation results. The improved LB formalism is then used to predict apparent permeability as a function of $K n$, allowing us to assess the reliability of the LB method against the $\mathrm{BKC}$ correlation, as well as other datasets.

\section{Methods}

The LB method describes the motion of gas particles statistically and simulates the spatiotemporal variation of particles' velocity distribution as governed by the discrete 
Boltzmann equation (Guo and Shu 2013). The distribution function $(f)$ represents the dimensionless mass density of particles with velocity $\boldsymbol{c}$ at location $\boldsymbol{x}$ and time $t$ (Krüger et al. 2017). Macroscopic gas properties, e.g., mass density, momentum density, total energy density, and flow velocity, can be calculated from weighted summations of the discrete velocity distribution.

a



b

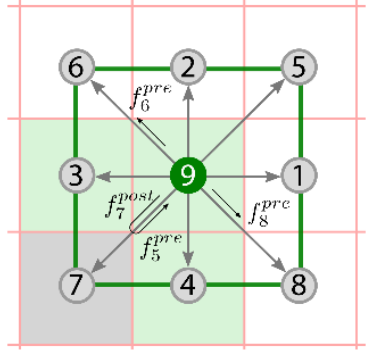

$c$

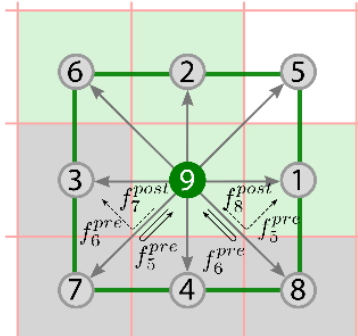

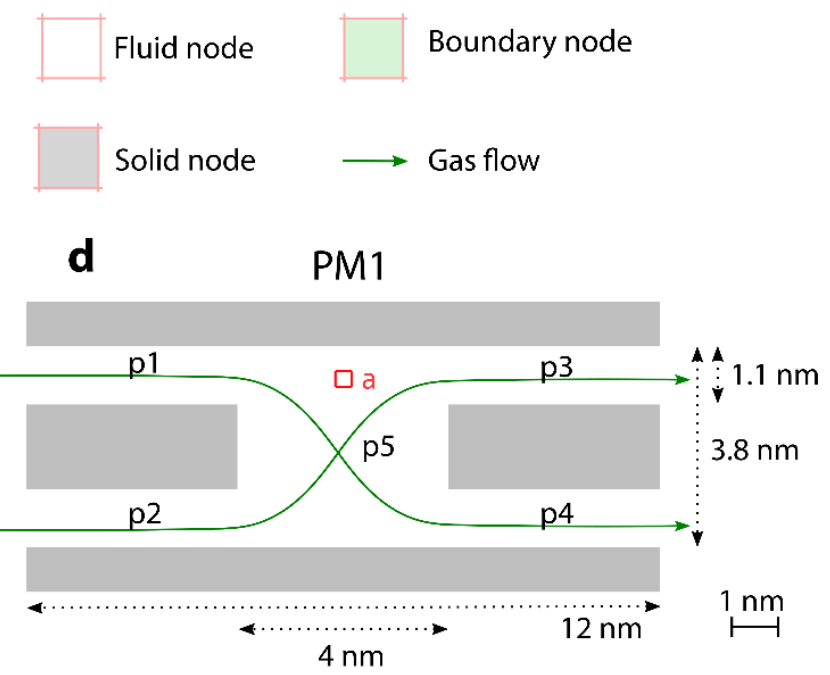

e $\quad \mathrm{PM} 2$

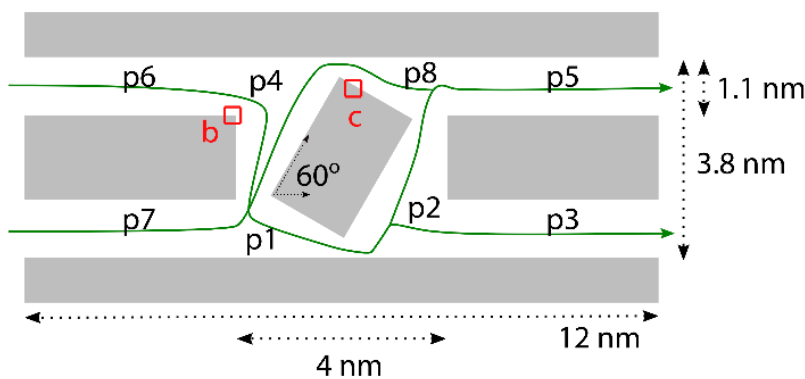

Fig. 1. Lattice Boltzmann models for gas flow through porous media (PM). (a) D2Q9 velocity sets. (b) the MBB BC. (c) the MBB+SR BC. (d) PM1 structure. (e) PM2 structure. Gray filled slabs are impermeable solid matrices. Void spaces are indexed pores (e.g., p1) with arrows denoting simulated flow directions. 


\subsection{Particle Collision and Propagation}

The LB model implemented in this work is based on a two-dimensional-nine-discretevelocity (D2Q9) set, shown schematically in Fig. 1a. Each square lattice is associated with nine nodes: Node 9 is at the center of the lattice site, and the others at the center of the neighboring lattice sites. Different nodes are assigned a different discrete velocity constant vector, i.e., $\boldsymbol{c}_{\alpha}^{*}=[(1,0),(0,-1),(-1,0),(0,1),(1,-1),(-1,-1),(-1,1),(1,1),(0,0)]$, for $\alpha=1,2, \ldots, 9$, respectively. Nodes are classified as fluid, boundary, and solid nodes, according to the location of the lattice site within the porous medium. As shown in Fig. 1b and 1c, the lattice sites in pore spaces are fluid nodes, in which the discrete Boltzmann equation applies. Solid nodes are representative of solid slabs, in which the velocity distribution remains zero. A boundary node, to which BCs applies, links a fluid and a solid node. We use 'the link-wise boundary node' where the computational boundary is located on the link between the physical boundary and fluid node.

If the central node (Node 9) is identified as a fluid or boundary node, particle collisions occur locally. The velocity distribution after a collision, denoted by $f_{\alpha}^{\text {post }}$, propagates along its discrete velocity vector $\left(\boldsymbol{c}_{\alpha}^{*}\right)$ to neighboring nodes. The propagated velocity distribution will be the velocity distribution before the next collision, denoted by $f_{\alpha}^{\text {pre }}$. One collision-propagation cycle occurs in a single time step, where each central lattice simultaneously receives $f_{\alpha}^{\text {post }}$ from and emits $f_{\alpha}^{\text {pre }}$ to its eight neighboring lattices. This process is governed by the discrete Boltzmann equation, Eq. (1), which models the change of velocity distributions during a collision at lattice location $\boldsymbol{x}^{*}$ and time $t^{*}$ (from $f_{\alpha}^{\text {pre }}$ to 
$f_{\alpha}^{\text {post }}$ on the right-hand-side (RHS)), and a particle propagation to the next lattice location $\boldsymbol{x}^{*}+\boldsymbol{c}_{\alpha}^{*} \delta t^{*}$ at the time $t^{*}+\delta t^{*}$ (from $f_{\alpha}^{p o s t}$ on RHS to $f_{\alpha}^{p r e}$ on the left-hand side).

$$
f_{\alpha}^{\text {pre }}\left(\boldsymbol{x}^{*}+\boldsymbol{c}_{\alpha}^{*} \delta t^{*}, t^{*}+\delta t^{*}\right)=\underbrace{f_{\alpha}^{\text {pre }}\left(\boldsymbol{x}^{*}, \boldsymbol{x}^{*}\right)+\Omega_{\alpha}\left(t^{*}\right)}_{f_{\alpha}^{\text {post }}\left(\boldsymbol{x}^{*}, t^{*}\right)}
$$

The superscript ' $*$ ' in Eq. (1) denotes non-dimensionalized parameters. The discrete collision operator $\Omega_{\alpha}$ models the velocity distribution relaxation to its equilibrium values ( $f_{\alpha}^{(e q)}$ ) at node $\alpha$. The linear Bhatnagar-Gross-Krook (BGK) collision operator is implemented, where $\Omega_{\alpha}=-\left(f_{\alpha}-f_{\alpha}^{(e q)}\right) /\left(\tau^{*}+0.5\right)$ and $\tau^{*}$ is the dimensionless relaxation time. Based on the Maxwell-Boltzmann distribution, the discrete equilibrium distribution for the isothermal condition can be expanded in the Hermite polynomials as (Krüger et al. 2017):

$$
f_{\alpha}^{(e q)}\left(\boldsymbol{x}^{*}, t^{*}\right)=w_{\alpha} \rho^{*}\left[1+\frac{c_{\alpha i}^{*} u_{i}^{*}}{\left(c_{s}^{*}\right)^{2}}+\frac{u_{i}^{*} u_{j}^{*}\left(c_{\alpha i}^{*} c_{\alpha j}^{*}-\left(c_{s}^{*}\right)^{2} \delta_{i j}\right)}{2\left(c_{s}^{*}\right)^{4}}\right] .
$$

In Eq. (2), subscripts $i, j$ denote a vector component, e.g., $u_{i}^{*} \in\left\{u_{x}^{*}, u_{y}^{*}\right\}$. The local fluid mass density $\rho^{*}$ can be calculated by local lattice velocity distributions in $\rho^{*}=\sum_{\alpha=1}^{q} f_{\alpha}$. The flow velocity $\boldsymbol{u}^{*}$ is calculated from the summation of velocity distributions in $\boldsymbol{u}^{*}=$ $\sum_{\alpha=1}^{q} \boldsymbol{c}_{\alpha}^{*} f_{\alpha} / \rho^{*}+0.5 \delta t^{*} \boldsymbol{a}^{*}$, where $\boldsymbol{a}^{*}$ is the dimensionless acceleration vector. $w_{\alpha}$ is a weighting factor for different nodes and $\sum_{\alpha=1}^{q} w_{\alpha}=1$. Within the D2Q9 velocity set, $q=$ $9, w_{9}=\frac{4}{9}, w_{1,2,3,4}=\frac{1}{9}$, and $w_{5,6,7,8}=\frac{1}{36}$. The non-equilibrium distribution $f_{\alpha}^{(n e q)}$, as the difference between $f_{\alpha}$ and $f_{\alpha}^{(e q)}$, reflects how far the fluid particles are from equilibria. 
Small for continuum flow, this value can be large for slip $(0.001<K n<0.1)$ and transitional flows $(0.1<K n<10)$.

When discretizing Eq. (1), the velocity space should be projected onto the Hermite space with orthonormal bases. The un-projected part of $f_{\alpha}$ will cause aliasing errors, which can be alleviated by projecting $f_{\alpha}$ onto the Hermite space by expanding $f_{\alpha}^{(n e q)}$ in the Hermite polynomials, named as 'the regularization' (Zhang et al. 2006). The projected (or

regularized) $f_{\alpha}^{(n e q)}$ is denoted by $\hat{f}_{\alpha}^{(n e q)}$. Up to the second-rank polynomials, $\hat{f}_{\alpha}^{(n e q)}$ reads

$$
\hat{f}_{\alpha}^{(n e q)}\left(\boldsymbol{x}^{*}, t^{*}\right)=w_{\alpha} \cdot \frac{\left(c_{\alpha i}^{*} c_{\alpha j}^{*}-\left(c_{s}^{*}\right)^{2} \delta_{i j}\right)}{2\left(c_{S}^{*}\right)^{4}} \sum_{\beta=1}^{q} f_{\beta}^{(n e q)}\left(\boldsymbol{x}^{*}, t^{*}\right)\left(c_{\beta i}^{*} c_{\beta j}^{*}-\left(c_{S}^{*}\right)^{2} \delta_{i j}\right)
$$

where $\hat{f}_{\beta}^{(n e q)}\left(\boldsymbol{x}^{*}, t^{*}\right) \approx f_{\beta}^{(n e q)}\left(\boldsymbol{x}^{*}, t^{*}\right)=f_{\beta}\left(\boldsymbol{x}^{*}, t^{*}\right)-f_{\beta}^{(e q)}\left(\boldsymbol{x}^{*}, t^{*}\right)$. The Kronecker delta $\delta_{i j}$ is 1 when $i=j$ and 0 when $i \neq j$.

\subsection{Knudsen Number and Relaxation Time}

Because the characteristic length $l$ is often considered as the hydraulic radius $\left(R_{H}\right)$, the Knudsen number in free space can be defined as (Civan 2010):

$$
K n=\frac{\lambda}{R_{H}}
$$

However, $R_{H}$ can be different for different pore geometries. In this work, gas flow is simulated through three porous matrixes (PM): PM1 (Fig. 1d), PM2 (Fig. 1e), and PM3 (Fig. 6a). We denote either the local pore height of PM1\&PM2 or the local pore diameter 
of PM3 as $H$. For PM1\&PM2, periodic boundary conditions are applied on the streamwise direction, and the pore geometries are treated as slit pores. In such a geometry, $R_{H}$ is approximated by the height of the pore, i.e., $R_{H}=H$ (Cengel 2010). For PM3, in which we apply non-periodic boundary conditions along the streamwise direction, the pore geometries are modeled as circular. For such geometry, $R_{H}$ is the quarter of the pore diameter, i.e., $R_{H}=H / 4$ (Cengel 2010).

To calculate $K n$ based on pore structure and gas properties, we use (Zhao et al. 2018)

$$
K n=\frac{k_{B} T}{\sqrt{2} \pi a_{0}^{2} P R_{H}}
$$

where $k_{B}$ is the Boltzmann constant; $P$ is gas pressure (assumed to equal the pore pressure) obtained by atomic number density of gas in the MD system and Peng-Robinson equation of state (Dahm and Visco 2014); $a_{0}$ is the fluid molecular diameter, approximated as the Lennard-Jones parameter $\sigma_{f f}$ (Bhatia and Nicholson 2006, Jasper and Miller 2014), e.g., for methane $\sigma_{f f} \approx 0.373 \mathrm{~nm}$.

In a confined space, the mean free path is shorter than in free space. The reduction factor is modeled as $\Psi(K n)=(1+b K n)^{-1}$ where $b$ is the Bosanquet parameter, typically $b=3.4$ (Kalarakis et al. 2012). A modified Knudsen number $K n^{\prime}=K n \Psi(K n)$ is used for PM1 and PM2.

The Knudsen number in PM3 includes the porosity $(\varepsilon)$ and the dimensionless permeability $(k)$ (Wu et al. 2017): 


$$
K n^{\prime \prime}=K n^{\prime} \sqrt{\frac{\varepsilon}{12 k}}
$$

where $k=\frac{k_{i n}}{H^{2} / 32}, k_{\text {in }}$ is the dimensional intrinsic permeability estimated by LB simulations, and the conversion factor $H^{2} / 32$ is the Darcy permeability for the tube (Lake 1989).

In the BGK collision operator in Eq. (1), the dimensionless relaxation time $\left(\tau^{*}\right)$ characterizes how soon particles will reach local equilibrium state from perturbation during collisions (Krüger et al. 2017, Succi and Succi 2018). $\tau^{*}$ relates to the dynamic viscosity ( $\eta$ ) in $\eta=c_{S}^{2} \rho \tau^{*} \delta t$, where $\rho$ is mass density. $c_{s}$ is the speed of sound, related to the universal gas constant $R$, molar mass $M$, and absolute temperature $T$ as $c_{s}=\sqrt{R T / M}$. In Equation (4), $R_{H}$ can be expressed in its dimensionless form as $R_{H}^{*}=R_{H} / \delta y$. The mean free path relates to the dynamic viscosity $(\eta)$ as $\lambda \approx \eta P^{-1} \sqrt{\pi R T / 2 M}$ (Guo and Shu 2013). Given that $c=\delta y / \delta t=\sqrt{\chi R T / M}$ (where $\chi$ is a constant, e.g. $\chi=3$ in the D2Q9 velocity set), $\tau^{*}$ is obtained as a function of $K n^{\prime}: \tau^{*}=\sqrt{2 \chi / \pi} R_{H}^{*} K n^{\prime}$.

\subsection{The Improved BC for Gas Slippage}

Since solid-gas interactions are reflected as molecular momentum exchanges, i.e., the exchange of $f_{\alpha}$ coming into, and going out from the fluid domain, BCs at the solid-gas interface may not only affect the flow behavior near the interface but also near the pore center. By imposing zero flow velocity in the direction of the surface normal but a nonzero velocity tangentially, the specular reflection (SR) BC yields infinite slip (Cornubert 
et al. 1991, Guo et al. 2007). The SR BC assumes that $f_{\alpha^{\prime}}^{\text {post }}$ of the outgoing $\boldsymbol{c}_{\alpha^{\prime}}$ is specularly reflected at the boundary. The reflected velocity is $\boldsymbol{c}_{\alpha}=\boldsymbol{c}_{\alpha^{\prime}}+2\left(\boldsymbol{c}_{\alpha} \cdot \boldsymbol{n}\right) \boldsymbol{n}$, where $\boldsymbol{c}_{\alpha} \cdot \boldsymbol{n}>0$ and $\boldsymbol{c}_{\alpha^{\prime}} \cdot \boldsymbol{n}<0$. The velocity distribution becomes

$$
f_{\alpha}^{\text {pre }}\left(\boldsymbol{x}^{*}, t^{*}+\delta t^{*}\right)=f_{\alpha^{\prime}}^{\text {post }}\left(\boldsymbol{x}^{*}, t^{*}\right)
$$

In Fig. 1c, $\boldsymbol{c}_{7}$ is reflected along the direction of $\boldsymbol{c}_{6}$ once hitting the bottom solid node, $f_{7}^{\text {post }}$ replaces $f_{6}^{\text {pre }}$ in a specular reflection. Similarly, $f_{8}^{\text {post }}$ replaces $f_{5}^{\text {pre }}$. In LB simulations of finite- $K n$ flow where gas slip is finite, a classical approach is to algebraically combine a no-slip BC (e.g., SBB) with a finite slip BC (e.g., SR). One can account for velocity distribution contributions from each $\mathrm{BC}$ via a combination fraction $(r)$ :

$$
f_{\alpha}^{p r e}\left(\boldsymbol{x}^{*}, t^{*}+\delta t^{*}\right)=r\left(\boldsymbol{x}^{*}\right) \cdot f_{\bar{\alpha}}^{\text {post }}\left(\boldsymbol{x}^{*}, t^{*}\right)+\left[1-r\left(\boldsymbol{x}^{*}\right)\right] \cdot f_{\alpha^{\prime}}^{\text {post }}\left(\boldsymbol{x}^{*}, t^{*}\right)
$$

The standard bounce-back (SBB) BC prescribes that at the boundary node, the outgoing velocity $\left(\boldsymbol{c}_{\bar{\alpha}}\right)$, once hitting the physical boundary, exchanges the velocity distribution by reversing its direction, i.e., the direction of the incoming velocity $\boldsymbol{c}_{\alpha}$. The incoming velocity distribution $\left(f_{\alpha}^{\text {pre }}\right)$ before the next collision is therefore assigned the outgoing velocity distribution $\left(f_{\bar{\alpha}}^{\text {post }}\right)$. That is,

$$
f_{\alpha}^{\text {pre }}\left(\boldsymbol{x}^{*}, t^{*}+\delta t^{*}\right)=f_{\bar{\alpha}}^{\text {post }}\left(\boldsymbol{x}^{*}, t^{*}\right)
$$


where $\boldsymbol{c}_{\alpha}=-\boldsymbol{c}_{\bar{\alpha}}, \boldsymbol{c}_{\alpha} \cdot \boldsymbol{n}>0, \boldsymbol{c}_{\bar{\alpha}} \cdot \boldsymbol{n}<0$, and $\boldsymbol{n}$ is the surface normal vector from solid to fluid. In Fig. $1 \mathrm{~b}, f_{7}^{\text {post }}$ of outgoing velocity $\boldsymbol{c}_{7}$ will replace $f_{5}^{\text {pre }}$ of the incoming velocity $\boldsymbol{c}_{5}$ before next collision. This velocity distribution inversion takes place only between an outgoing velocity and an incoming velocity, in pairs. The rest of the discrete velocities considered in our model are parallel to the boundary node, where $\boldsymbol{c}_{\alpha} \cdot \boldsymbol{n}=0$, i.e., $\boldsymbol{c}_{6}$ and $\boldsymbol{c}_{8}, \boldsymbol{c}_{2}$ and $\boldsymbol{c}_{4}, \boldsymbol{c}_{3}$ and $\boldsymbol{c}_{1}$. In the SBB scheme, parallel velocities will not exchange their velocity distribution by reversing their direction, but instead by propagating velocity distributions from neighboring fluid and boundary nodes instead (Guo and Shu 2013).

The correct implementation of the combined BC relies on the prerequisite that the noslip BC can achieve a physically sound no-slip condition. However, the analytical solutions reveal that SBB is insufficient to mirror a no-slip boundary (Meng et al. 2018) since the 'uncontrolled' parallel velocity sets can lead to unphysical slip. When parallel velocities propagate, they are subject to acceleration, pressure, and fluid density at inlet and outlet boundaries, which may cause a tangential non-zero velocity distribution, and therefore lead to unphysical slip. A modified bounce back (MBB) BC is needed to enforce zero slip. One approach is to impose that each pair of parallel velocity distributions $\boldsymbol{c}_{\alpha 1}$ and $\boldsymbol{c}_{\alpha 2}$ is equivalent after the collision (Meng et al. 2018):

$$
f_{\alpha 1}^{\text {pre }}\left(\boldsymbol{x}^{*}, t^{*}+\delta t^{*}\right)=0.5\left(f_{\alpha 1}^{\text {post }}\left(\boldsymbol{x}^{*}, t^{*}\right)+f_{\alpha 2}^{\text {post }}\left(\boldsymbol{x}^{*}, t^{*}\right)\right)
$$

and 


$$
f_{\alpha 2}^{\text {pre }}\left(\boldsymbol{x}^{*}, t^{*}+\delta t^{*}\right)=0.5\left(f_{\alpha 1}^{\text {post }}\left(\boldsymbol{x}^{*}, t^{*}\right)+f_{\alpha 2}^{\text {post }}\left(\boldsymbol{x}^{*}, t^{*}\right)\right) \text {. }
$$

When handling the parallel velocities, this approach requires identifying the parallel velocities at the boundary, which can be tedious in complex PMs. Unfortunately, not implementing this approach can negatively affect the accuracy of the results, as discussed in Section 0.

Here the $\mathrm{SBB}+\mathrm{SR} \mathrm{BC}$ is improved by combining $\mathrm{MBB}$ and $\mathrm{SR} \mathrm{BCs}$ to avoid the unphysical slip in the SBB scheme while manageable computational costs for conducting LB simulations. Specifically, we maintain the SR scheme (Eq. (7)) and the combination rule (Eq. (8)) and impose the equivalence of parallel velocities in pairs for the bounce-back scheme (Eqs. (9) through (11)).

To realize the second-order slip in LB simulations, we adopted the following formula for the combination fraction $r$ (Guo et al. 2007):

$$
r=\left\{1+\sqrt{\frac{\pi}{6}}\left[\frac{1}{4 K n^{*}\left(R_{H}^{*}\right)^{2}}+A_{1}+\left(2 A_{2}-\frac{8}{\pi}\right) K n^{*}\right]\right\}^{-1}
$$

Equation (9) was originally derived for the classical BC inclusive of standard bounce-back and specular reflection, and it holds also for the proposed BC. In deriving Equation (9), as shown by others (Guo et al. 2007), all velocity distributions $\left(f_{i}\right)$ including the parallel velocities are lumped into the streamwise velocity $(u)$. The derived expression of $u$ is applied to the finite-difference equation to obtain the formula of slip velocity in LB 
simulations, and the derived LB slip velocity is then compared with the analytical slip velocity to derive the expression of $r$ as in Equation (9).

According to the second-order slip condition, the slip velocity $\left(u_{s}\right)$ can be expressed as

$$
u_{s}=A_{1} \lambda\left(\frac{\partial u}{\partial \mathbf{n}}\right)_{\text {wall }}-A_{2} \lambda^{2}\left(\frac{\partial^{2} u}{\partial \mathbf{n}^{2}}\right)_{\text {wall }}
$$

where coefficients $A_{1}=(2-\sigma)(1-0.1817 \sigma) / \sigma$ and $A_{2}=\pi^{-1}+0.5 A_{1}^{2}$. The tangential momentum accommodation coefficient (TMAC), denoted by $\sigma$, is a measurement of solidgas interactions and corresponds to an average exchange rate between the incident and reflected tangential momentum when gas molecules hit the wall, i.e., $\sigma=\left(\tau_{R}-\right.$ $\left.\tau_{I}\right) /\left(\tau_{\text {wall }}-\tau_{I}\right)$, where $\tau_{R}, \tau_{I}$, and $\tau_{\text {wall }}$ are the tangential momentum of reflected molecules, incident molecules, and wall surface, respectively (Di Staso 2018). When $\sigma=$ 0 , specular reflection is present; when $\sigma=1$, full diffuse reflection occurs. In LB models, TMAC is often chosen to be unity ( $\mathrm{Li}$ et al. 2018, Wang et al. 2017, Yu et al. 2017), which assumes full diffuse reflection. However, a recent numerical study indicates that TMAC should be non-unitary in porous media (Wu et al. 2017). Experiments also show that TMAC values for Helium, Argon, and Nitrogen gases are not unitary in second-order slip simulations (Zhang et al. 2012). Since the experimental TMAC for methane is not available, we assume the value reported for Nitrogen gas $(\sigma \approx 0.827)$ as the value for methane in our LB simulations (Yamaguchi et al. 2012). 


\subsection{Gas Permeability of Pressure-Driven Flow}

We simulate gas flow through PM driven by a pressure gradient across the inlet and the outlet of the PM, where the external force $\boldsymbol{F}_{\alpha}^{*}$ is added to Eq. (1). After being combined with Eqs. (2) and (3), Eq. (1) becomes

$$
f_{\alpha}^{p r e}\left(\boldsymbol{x}^{*}+\boldsymbol{c}_{\alpha}^{*} \delta t^{*}, t^{*}+\delta t^{*}\right)=\frac{2 \tau^{*}-1}{2 \tau^{*}+1} \hat{f}_{\alpha}^{(n e q)}\left(\boldsymbol{x}^{*}, t^{*}\right)+f_{\alpha}^{(e q)}\left(\boldsymbol{x}^{*}, t^{*}\right)+\delta t^{*} \boldsymbol{F}_{\alpha}^{*},
$$

where $\boldsymbol{F}_{\alpha}^{*}$ is discretized as:

$$
F_{\alpha i}^{*}=\frac{2 \tau^{*}}{2 \tau^{*}+1} w_{\alpha} \rho^{*}\left[\frac{c_{\alpha i}^{*} a_{i}^{*}}{\left(c_{S}^{*}\right)^{2}}+\frac{a_{i}^{*} u_{j}^{*}\left(c_{\alpha i}^{*} c_{\alpha j}^{*}-\left(c_{s}^{*}\right)^{2} \delta_{i j}\right)}{\left(c_{S}^{*}\right)^{4}}\right] .
$$

In Eq. (15), $F_{\alpha i}^{*}$ and $a_{i}^{*}$ are the component of the force vector $\boldsymbol{F}_{\alpha}^{*}$ and the acceleration vector $\boldsymbol{a}^{*}$, respectively. The acceleration is related to force density $\left(\boldsymbol{F}_{v}^{*}\right)$ in terms of fluid mass density $\left(\rho^{*}\right): \boldsymbol{F}_{v}^{*}=\rho^{*} \boldsymbol{a}^{*}$. The force density equals the pressure gradient in the opposite direction, as $F_{v}^{*}=-\nabla P^{*}$. Based on Darcy's law, directional permeability in PM is estimated by average fluid velocity under a pressure gradient: $k_{i}^{*}=-\overline{u_{i}^{*}} \overline{\eta^{*}} / \nabla P_{i}^{*}$, where $i$ indicates the direction of the measured permeability. $\overline{\eta^{*}}$ is the average dynamic viscosity, which is calculated by $\overline{\eta^{*}}=\rho^{*}\left(c_{s}^{*}\right)^{2} \overline{\tau^{*}}$, where $\overline{\tau^{*}}$ is the average relaxation time within different pores. Based on the above formulae, permeability is estimated by $k_{i}^{*}=$ $\overline{u^{*}}{ }_{i}\left(c_{s}^{*}\right)^{2} \overline{\tau^{*}} / a_{i}^{*}$ (dimensionless) and $k_{i}=C_{x}^{2} k_{i}^{*}$ (dimensional), where $C_{x}$ is the unit conversion factor $\left(C_{l}\right)$ for length in Table 1. 


\subsection{Model Setup}

Input data in the LB simulations are summarized in Table 1. The fluid kinematic viscosity determines the simulation time step $\delta t$. Unit conversion from the dimensionless LB environment to the physical system, tabulated in Table 2, is essential for data analysis. In our study, the unit conversion factor for the time $C_{t}$ is obtained by the conversion of kinematic viscosity $(v): \eta=\rho\left(c_{s}^{*}\right)^{2} \tau^{*} C_{l}^{2} / C_{t}$. To obtain a constant value of $C_{t}$, we consider that the local $\eta$ in a pore is proportional to its $\tau^{*}: \eta / \eta_{0}=\tau^{*} / \tau_{0}^{*}$, where the subscript ' 0 ' denotes the value in the reference pore with a height of $1.1 \mathrm{~nm}$. The value of $\eta_{0}$ is obtained from the bulk velocity profile in MD results, which is consistent with data from Ref. (Lemmon et al. 2019).

The gas density from MD is used to estimate fluid pressure and $K n^{\prime}$. Local $K n^{\prime}$ is calculated for each pore, followed by the calculation of $\tau^{*}$ in Section 2.2 and $r$ as a function of $K n^{\prime}$ in Eq. (12). Solid-gas interactions are modeled by specifying the TMAC, as described in Section 2.2.

To initiate LB simulations, structural information of porous media is required including pore diameter, dimensions of the PM, and locations of boundary nodes. In Figs. 1d and 1e, we show the structures of the porous media used in this work, denoted by PM1 and PM2. In PM1, p1 through p4 are congruent micropores where the height $H=1.1 \mathrm{~nm}$ and the pore length $L=4 \mathrm{~nm}$; p5 is a mesopore with $H=3.8 \mathrm{~nm}$ and $L=4 \mathrm{~nm}$. In PM2, p1 \& p8, p4 \& p2, p3 \& p5 through p7 are micropores congruent in pairs. Two PM share a similar structure expect a mesopore in PM1 (p5) is subdivided into four micropores in PM2 
(i.e., p1, p2, p4, p8) by a tilted slab with $60^{\circ}$ rotation. Periodic boundary conditions are set on the left and right end of PM1 and PM2.

Uniform square lattices of the side $\delta x$ are set up according to the specified spatial resolution. Eq. (14) along with the classical (i.e., SBB and SBB+SR) BCs and the improved (i.e., $\mathrm{MBB}$ and $\mathrm{MBB}+\mathrm{SR}$ ) $\mathrm{BCs}$ is applied to predict gas transport behavior in micro-/nanoporous media when the flow reaches a steady state. We impose bounce-back BCs (where $r=1$ in Eq. (8)) and $\tau^{*}=1$ to simulate intrinsic permeability $\left(k_{i n}\right)$ and slip BCs for apparent gas permeability. Lastly, we evaluate the Klinkenberg effect by calculating the permeability correction factor, i.e., the ratio of apparent to intrinsic permeability $\left(k_{\text {app }} / k_{\text {in }}\right)$.

Table 1. Input data for LB simulations.

\begin{tabular}{|c|c|c|c|c|}
\hline & \multicolumn{2}{|l|}{ PM1 } & \multirow{2}{*}{$\begin{array}{l}\text { PM2 } \\
\text { p1 through p8 }\end{array}$} & \multirow{2}{*}{ Source } \\
\hline & p1 through p4 & $\mathrm{p} 5$ & & \\
\hline$n_{0}\left(1 / \AA^{3}\right)$ & 0.0058 & 0.0043 & 0.0055 & MD simulations \\
\hline$\eta(\mathrm{Pa} \cdot \mathrm{s})$ & $2.20 \mathrm{e}-05^{\mathrm{a}}$ & $4.30 \mathrm{e}-05^{\mathrm{b}}$ & $1.90 \mathrm{e}-05^{\mathrm{c}}$ & MD simulations \\
\hline$\nabla P(\mathrm{MPa} / \mathrm{nm})$ & 8.060 & 5.975 & 7.643 & $\nabla P=-\boldsymbol{F}_{\text {ext }} n_{0}$ \\
\hline TMAC & 0.827 & 0.827 & 0.827 & (Zhang et al. 2012) \\
\hline
\end{tabular}

a the reference dynamic viscosity $\eta_{0}$ in PM1.

${ }^{\mathrm{b}}$ calculated by $\eta=\tau^{*} \eta_{0} / \tau_{0}^{*}$.

${ }^{\mathrm{c}}$ the reference dynamic viscosity $\eta_{0}$ in PM2.

To validate the effectiveness of the improved BCs in simulating finite- $K n$ flow, we conducted nonequilibrium MD simulations of methane transport through PM1 and PM2. Information regarding the MD models is summarized in the supplementary information. In 
LB simulations, the pressure gradient $-\nabla P$ equals to the force density $\left(\boldsymbol{F}_{v}=\rho \boldsymbol{a}\right)$. In MD, to simulate a pressure-driven flow, each molecule is subject to a constant external force $\left(\boldsymbol{F}_{\text {ext }}\right)$, where $\boldsymbol{F}_{\text {ext }}=\boldsymbol{F}_{v} / n$ (Obliger et al. 2016), where $n$ is the atomic number density, $n=\rho N_{A} / M$. Therefore, the acceleration term is expressed in $\boldsymbol{a}=\boldsymbol{F}_{\text {ext }} N_{A} / M$. To compare LB and atomistic MD simulations, the unit conversions for velocity and acceleration are performed based on the unit conversions of time and length in Table 2 (Horbach and Succi 2006, Krüger et al. 2017).

In supporting information, we show the discrete effect on the improved BCs and the convergence of the estimated permeability with time steps. The robustness of the improved BCs with low-resolution PM structure images is discussed.

Table 2. Unit conversion between dimensional and the dimensionless LB systems (Krüger et al. 2017).

\begin{tabular}{|c|c|c|}
\hline & Dimensional (units) & Dimensionless \\
\hline Length & $L(\mathrm{~m})$ & $L^{*}\left(\mathrm{lu}^{\mathrm{a}}\right)$ \\
\hline Hydraulic radius & $R_{H}(\mathrm{~m})$ & $R_{H}^{*}(\mathrm{lu})$ \\
\hline Lattice side & $\delta x$ or $\delta y(\mathrm{~m})$ & $\delta x^{*}=\delta y^{*}=1(\mathrm{lu})$ \\
\hline Elapsed time & $t(\mathrm{~s})$ & $t^{*}\left(\mathrm{ts}^{\mathrm{b}}\right)$ \\
\hline Time step & $\delta t(\mathrm{~s})$ & $\delta t^{*}=1(\mathrm{ts})$ \\
\hline Lattice velocity & $c=\frac{\delta x}{\delta t}=\sqrt{\frac{\chi R T}{M}}(\mathrm{~m} / \mathrm{s})$ & $c^{*}=\frac{\delta x^{*}}{\delta t^{*}}=1(\mathrm{lu} / \mathrm{ts})$ \\
\hline The conversion factor for distance & $C_{l}=\frac{L}{L^{*}}=\frac{R_{H}}{R_{H}{ }^{*}}=\frac{\delta x}{\delta x^{*}}=\frac{\delta y}{\delta y^{*}}(\mathrm{~m})$ & \\
\hline The conversion factor for time & $C_{t}=\frac{t}{t^{*}}=\frac{\delta t}{\delta t^{*}}(\mathrm{~s})$ & \\
\hline The conversion factor for velocity & $C_{u}=\frac{C_{x}}{C_{t}}=\frac{u_{x}}{u_{x}^{*}}(\mathrm{~m} / \mathrm{s})$ & \\
\hline The conversion factor for acceleration & $C_{a}=\frac{C_{x}}{C_{t}^{2}}=\frac{a}{a^{*}}\left(\mathrm{~m}^{2} / \mathrm{s}\right)$ & \\
\hline
\end{tabular}





\section{Dimensionless streamwise velocity normalized by bulk velocity \\ e f \\ g}

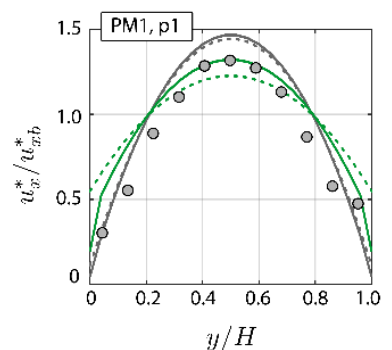

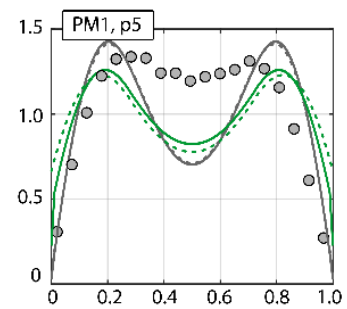

$y / H$

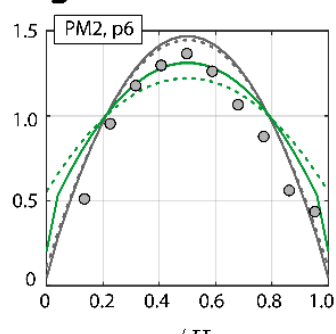

h



Fig. 2. Flow velocity profile. (a) through (d), LB results of dimensional streamwise velocity profiles in specified pores. (e) through (h), LB results of dimensionless streamwise velocity profiles are normalized by the dimensionless local bulk velocity. See text for a description of the figure.

\section{Results}

\subsection{Flow Velocity from LB and MD Simulations}

In Fig. 2, we present streamwise velocities obtained from LB and MD simulations for gas flowing through PM1 and PM2. Figs. 2a through 2d show dimensional streamwise ( $x$ directional) velocity profiles along the $y$-direction within pores $\mathrm{p} 1$ and $\mathrm{p} 5$ of PM1, and $\mathrm{p} 6$, p4, p1 of PM2, respectively. This figure allows us to compare LB results when slip (SBB+SR and $\mathrm{MBB}+\mathrm{SR}$ ) and no-slip (SBB and $\mathrm{MBB}$ ) BCs are implemented. Based on gas density and pore structure in MD models, average $K n^{\prime}=0.132$ for PM1 and 0.155 for 
PM2 are used for LB simulations. Different BCs are compared. Under the MBB+SR BC, the Mach number is calculated as $M a=\overline{u_{x}^{*}} / c_{s}^{*}=0.0505$ for PM1 and 0.0236 for PM2.

Visual analysis of the LB results confirms that different BCs yield distinctive flow behaviors. Comparing the LB results when no-slip BCs are implemented, we observe that the MBB BC yields lower slip than the SBB BC. Approaching the pore center, the difference between predictions by MBB and SBB BCs becomes more discernible. This implies that errors of numerical slip, if any, at the wall affect the prediction of flow velocity in the whole pore.

Compared to the MD predictions, the $\mathrm{LB}$ results obtained with the $\mathrm{MBB}+\mathrm{SR} \mathrm{BC}$ yield better match than the SBB+SR BC, both near the wall and in the pore centers. Such a difference is more evident than the difference when the MBB and the SBB BCs are applied. This highlights the importance weight of the bounce-back scheme in the combined $\mathrm{BB}+\mathrm{SR}$ BCs during LB simulations. Some small deviations are observed between MD and LB results obtained when implementing the $\mathrm{MBB}+\mathrm{SR} \mathrm{BC}$ because our $\mathrm{LB}$ representation does not account for surface roughness as MD models do. When the SBB+SR BC is implemented, the LB velocity profiles overestimate the MD results across the whole pore, especially in the pore of $1.1 \mathrm{~nm}$ height.

In Figs. $2 \mathrm{e}$ through $2 \mathrm{~h}$, the profiles of the normalized dimensionless velocity $\left(u_{x}^{*}\right)$ by the local 'bulk' velocity $\left(u_{x b}^{*}=\int_{0}^{H^{*}} u_{x}^{*} d y^{*} / H^{*}\right)$ are presented. Visual analysis reveals that velocity normalization has the effect of minimizing the differences observed when different $\mathrm{BCs}$ are implemented: evaluating the performance of the $\mathrm{BC}$ in $\mathrm{LB}$ simulations against $\mathrm{MD}$ 
results therefore becomes difficult and misleading. For example, in both PM1 and PM2, implementations of no-slip boundaries, i.e., $\mathrm{MBB}$ and $\mathrm{SBB}$, seem to reproduce MD results, which is, however, not physical for gas slippage. Significant discrepancies are observed between $\mathrm{LB}$ results obtained by the $\mathrm{MBB}+\mathrm{SR} \mathrm{BC}$ and $\mathrm{MD}$ results near the pore center in p5 of PM1 (Fig. 2f), which also indicates that the normalized velocity, although commonly applied to validate LB results, may not be as appropriate as the dimensional velocity is for the identification of the correct BC.
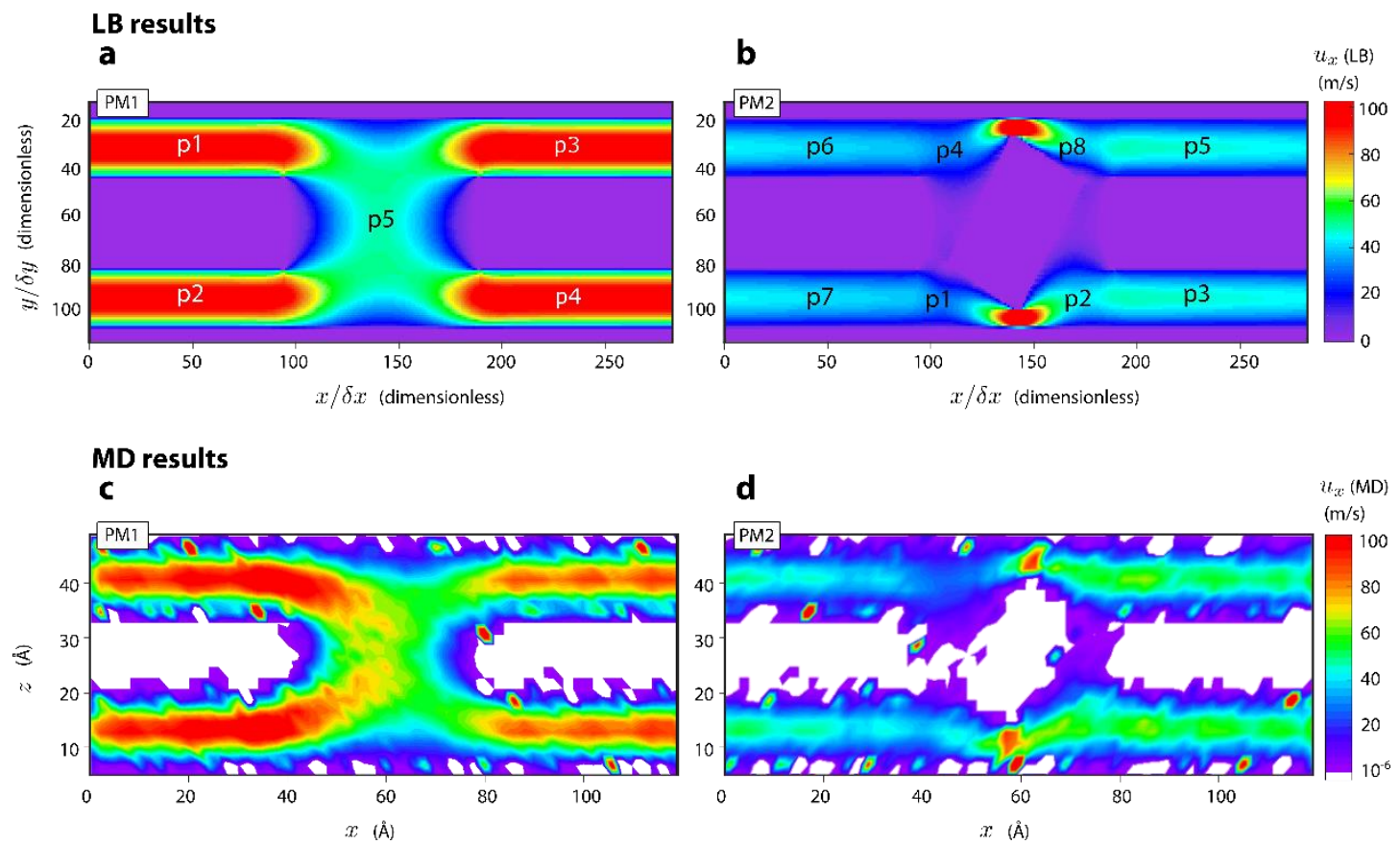

Fig. 3. Flow velocity distribution results of PM1 and PM2 in LB: (a) \& (b), and MD: (c) \& (d). White slabs in MD results represent the regions occupied by the amorphous silica substrates.

The velocity distributions in the streamwise direction are presented in Figs. $\mathbf{3 a}$ and $\mathbf{3 b}$ (LB simulations with the MBB+SR BC), and Figs. 3c and (MD simulations). We observe 
that the velocity distributions obtained from LB and MD simulations compare very well, in general. The average streamwise velocities in PM1 are $60.88 \mathrm{~m} / \mathrm{s}$ and $57.10 \mathrm{~m} / \mathrm{s}$ in LB and MD simulations, respectively, with a relative difference of $-7 \%$. LB velocity distributions are smoother than MD ones, as expected. This is because LB results show the statistical variation of velocity instead of the velocity of individual molecules as in MD results. Another finding is that the LB simulation for PM1 yields a symmetric flow velocity distribution (Fig. 3a) while MD results show a somewhat less symmetric flow behavior. This is because we approximate the same bulk gas density in all micropores of PM1 in the LB simulations, while in MD simulations, we observe more gas molecules accumulated in the inlet of the micropore p3 and p4 due to an entropy barrier (Phan and Striolo 2019), which reduces the average velocity of gas molecules in downstream of the mesopore p5.

In PM2, LB and MD results (Figs. $3 \mathrm{~b}$ and $\mathbf{3 d}$ ) are locally and globally consistent. Average streamwise velocities are $28.61 \mathrm{~m} / \mathrm{s}$ and $29.50 \mathrm{~m} / \mathrm{s}$ in $\mathrm{LB}$ and MD results, respectively, with a relative difference of $3 \%$. As the pore structure in PM2 includes a tilted solid rectangular obstacle in the middle, asymmetric velocity distributions are expected. In highly constricted spaces between $\mathrm{p} 4$ and $\mathrm{p} 8$, as well as $\mathrm{p} 1$ and $\mathrm{p} 2$, velocity maxima are observed in both MD and LB results. Comparing the velocities in those constricted areas indicates that LB simulations yield a higher flow velocity than MD. This is possibly due to the slightly different morphology of the solid substrate. In the MD models, we model amorphous silica, the surface of which causes some local backflow near the surface sites where gas molecules are bounced back in the direction opposite to the main streamwise, which reduces the average values of gas molecules velocity near the surface. 


$$
k_{i n}\left\{\begin{array} { l l } 
{ \Delta } & { \mathrm { LB } ( \mathrm { SBB } ) } \\
{ \square } & { \mathrm { LB } ( \mathrm { MBB } ) }
\end{array} \quad k _ { a p p } \left\{\begin{array}{ll}
\circ & \mathrm{MD} \text { data } \\
\Delta & \mathrm{LB}(\mathrm{SBB}+\mathrm{SR}) \\
\square & \mathrm{LB}(\mathrm{MBB}+\mathrm{SR})
\end{array}\right.\right.
$$

a
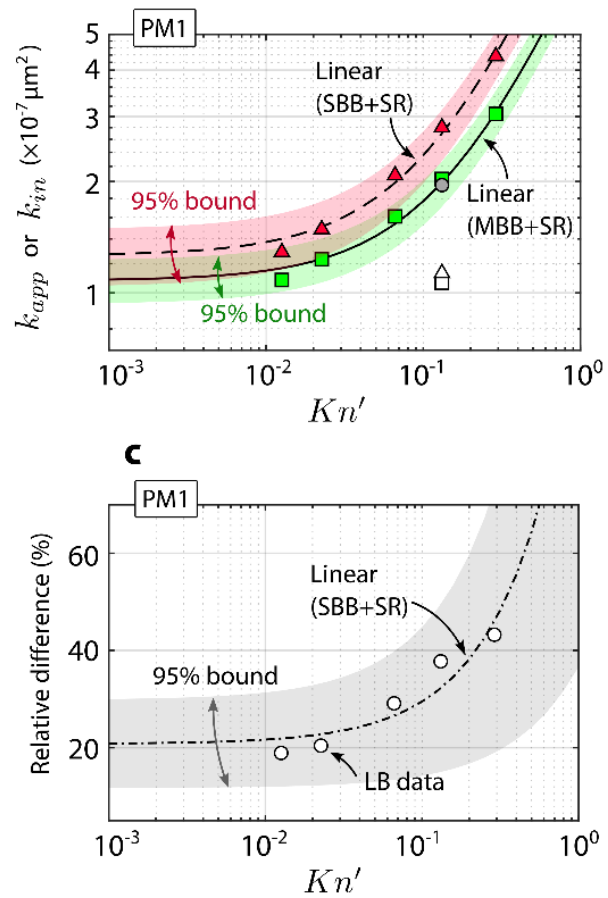

b

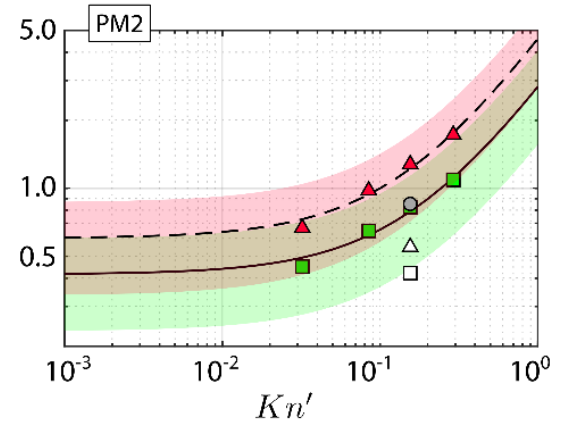

d

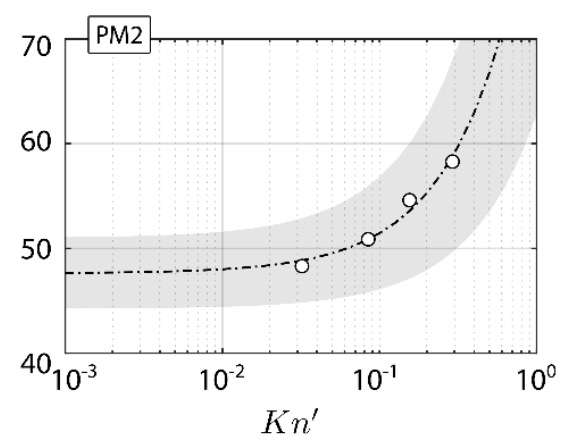

Fig. 4. Permeability estimates from MD and LB simulations with different BCs. (a) \& (b), comparisons of $k_{\text {in }}$ and $k_{\text {app }}$ estimated by the MD and LB simulations with different BCs. (c) \& (d), the relative difference of the estimated apparent permeability by SBB and MBB BCs as a linear relation of $K n^{\prime}$ where the relative difference is calculated by $\left(k_{a p p}^{(S B B+S R)}-k_{a p p}^{(M B B+S R)}\right) / k_{a p p}^{(M B B+S R)} \times 100 \%$.

\subsection{Gas Permeability and Klinkenberg Effect}

Figs. $4 \mathrm{a}$ and $\mathbf{4 b}$ summarize permeability estimates from LB and MD simulations at the average $K n^{\prime}=0.132$ in PM1 and 0.155 in PM2, respectively. The $k_{a p p}$ predicted for PM1 (Fig. 4a) is $1.957 \mathrm{e}-07 \mu \mathrm{m}^{2}$ (MD), 2.033e-07 $\mu \mathrm{m}^{2}$ (LB with the MBB+SR BC), and 2.801e$07 \mu \mathrm{m}^{2}$ (LB with the SBB+SR BC). The relative difference between LB and MD results is $-4 \%$ and $43 \%$ by the $\mathrm{MBB}+\mathrm{SR}$ and the $\mathrm{SBB}+\mathrm{SR} \mathrm{BCs}$, respectively. We estimate $k_{\text {in }}$ as $1.065 \mathrm{e}-07 \mu \mathrm{m}^{2}$ and $1.131 \mathrm{e}-07 \mu \mathrm{m}^{2}$ by implementing the MBB and SBB BCs, respectively, 
which gives a relative difference of $6 \%$. Because of computing power limitations, we cannot obtain intrinsic permeability data from MD simulations at $K n^{\prime} \rightarrow 0$. Permeability results for PM2 are presented in Fig. 4b.

We conducted a series of LB simulations to study the variation in $k_{a p p}$ as a function of $K n^{\prime}$, where different gas densities are used. The results are shown in Fig. 4a for PM1. Through linear regression, we find the estimated apparent permeability increases with $K n^{\prime}$ linearly with statistical accuracy $R^{2}=0.9938$ and 0.9942 by the MBB+SR BC and the $\mathrm{SBB}+\mathrm{SR} \mathrm{BC}$, respectively. The linear relations can be found in the supplementary information. As shown in Fig. $4 \mathrm{c}$, the relative differences between $k_{a p p}^{(S B B+S R)}$ and $k_{a p p}^{(M B B+S R)}$ show a linear relation with $K n^{\prime}$. Similar trends are found for PM2, as shown in Figs. $4 \mathrm{~b}$ and $4 \mathrm{~d}$. 

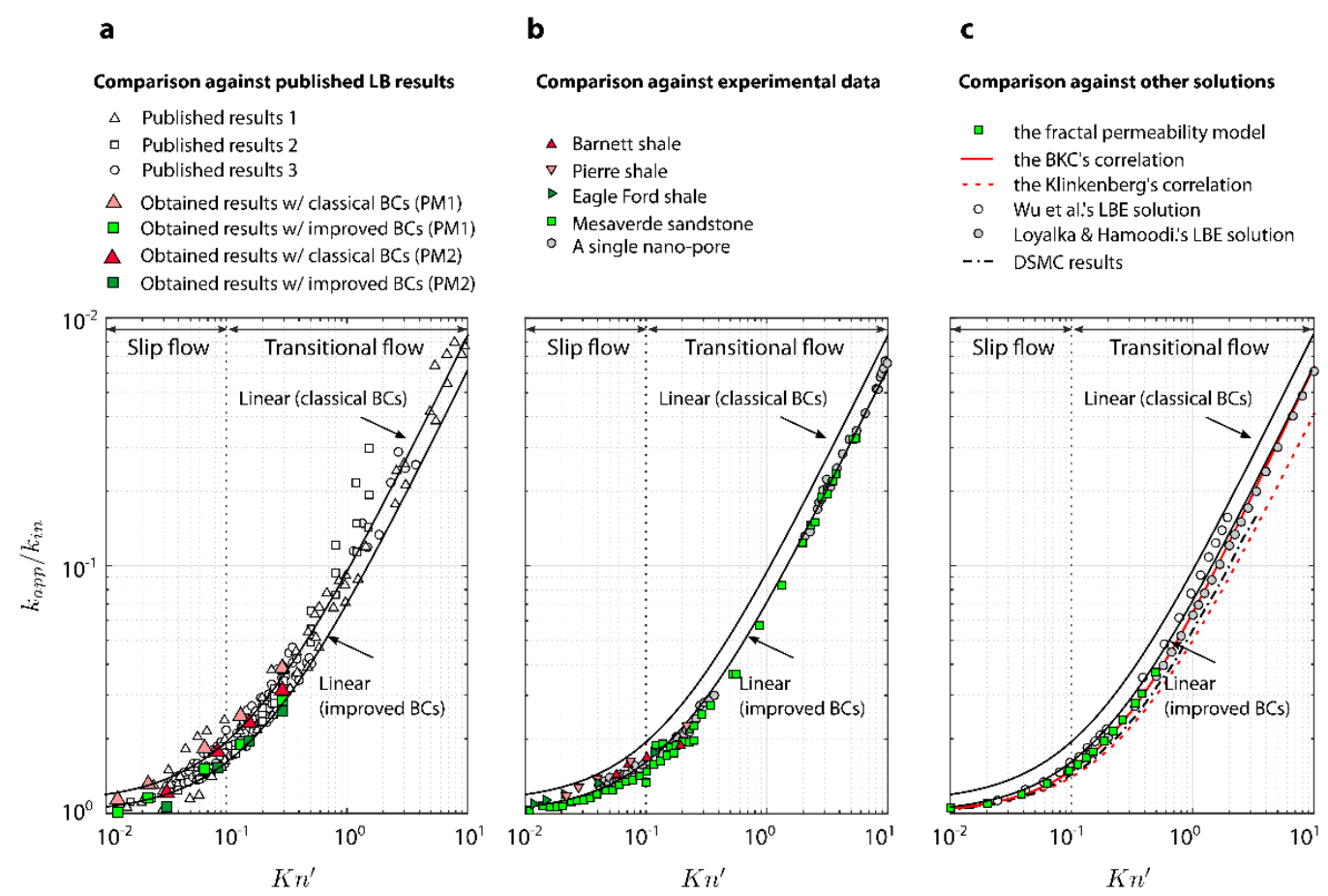

Fig. 5. LB results of $k_{a p p} / k_{\text {in }}$ obtained in this work using classical BCs and the improved one proposed here. (a) compared against published LB results using classical BCs: results 1 (Zhao and Wang 2019), results 2 ( $\mathrm{Li}$ et al. 2018), and results 3 (Chen et al. 2015). (b) compared against experimental data: shales (Moghaddam and Jamiolahmady 2016), tight sandstones (Ziarani and Aguilera 2012), and a single nano-pore (Velasco et al. 2012). (c) compared against predictions from non-LB approaches: 1) the fractal permeability with the second-order gas slip (Song et al. 2018); 2) the BKC's correlation (Beskok and Karniadakis 1999, Klinkenberg 1941); 4) LBE solutions (Loyalka and Hamoodi 1990, Wu et al. 2017); 5) DSMC results (Borner et al. 2017).

In Fig. 5a, we compare the Klinkenberg effect as predicted by the LB simulations that implement the $\mathrm{MBB}+\mathrm{SR}$ and $\mathrm{SBB}+\mathrm{SR} \mathrm{BC}$. In this figure, the permeability correction factor $\left(k_{a p p} / k_{\text {in }}\right)$ is plotted against $K n^{\prime}$. Numerical results predicted by the classical BCs are higher than those by the improved BCs when $0.013 \leq K n^{\prime} \leq 0.291$. Due to the wall effect, the $K n^{\prime}$ will reach an upper limit (e.g., $\approx 0.29$ for $b=0.34$ ) beyond which no more $k_{a p p} / k_{\text {in }}$ values are obtainable (Kalarakis et al. 2012). To predict $k_{a p p} / k_{\text {in }}$ in a broader 
$K n^{\prime}$ range, linear regressions are performed, shown in Fig. 5a. These relations are duplicated in Figs. $\mathbf{5 b}$ and $\mathbf{5 c}$ for further comparisons.

In Fig. 5a, we also compare selected LB results from literature (Chen et al. 2015, Li et al. 2018, Zhao and Wang 2019). These studies either implemented the SBB+SR BC, or applied the SBB BC first to estimate $k_{\text {in }}$ and then the second-order slip analytical solution to estimate $k_{a p p}$. Albeit scattered over a broad range, those results converge to our linear regression for the classical $\mathrm{BCs}$ when $0.1 \leq K n^{\prime} \leq 10$. In contrast, the linear relation predicted by the improved $\mathrm{BCs}$ indicates lower $k_{a p p} / k_{\text {in }}$ values. Fig. $5 \mathrm{~b}$ shows the trend of $k_{a p p} / k_{\text {in }}$ versus $K n^{\prime}$ reported from core-flooding experiments. Our improved LB BC yields a better match with the consistent trend from the sampled data than the classical BCs do.

In Fig. 5c, we compare the LB results against some recent non-LB approaches including fractal permeability models, empirical correlations, and numerical solutions (i.e., solutions of the linearized Boltzmann equation (LBE) and the direct simulation Monte Carlo (DSMC)). Most of the results presented were obtained using Argon and Nitrogen gases as the carrier fluid. We applied a TMAC of 0.827 in our LB simulations, which is in the range of TMAC values for Argon and Nitrogen gases (Yamaguchi et al. 2017, Zhang et al. 2012). We find that when $0.01 \leq K n^{\prime} \leq 10$, the fractal permeability model, the BKC correlation, and solutions of the linearized Boltzmann equation agree with the linear relation predicted by the improved $\mathrm{BC}$. Other models, i.e., DSMC and the Klinkenberg's correction, are found to predict lower values than the linear relation for different reasons. Firstly, our LB 
simulations simulate the flow at the temperature of $338 \mathrm{~K}$ while the presented DSMC results were obtained at $310 \mathrm{~K}$. The DSMC results imply that the Klinkenberg effect decreases as temperature decreases (Borner et al. 2017). Secondly, our LB simulations predict the second-order slip while the Klinkenberg's correlation assumes first-order slip (Ghanbarian and Javadpour 2017). Studies find that the first-order slip assumption fails in the transitional flow region where $K n^{\prime} \geq 0.1$ (Hadjiconstantinou 2005, Hadjiconstantinou 2006).
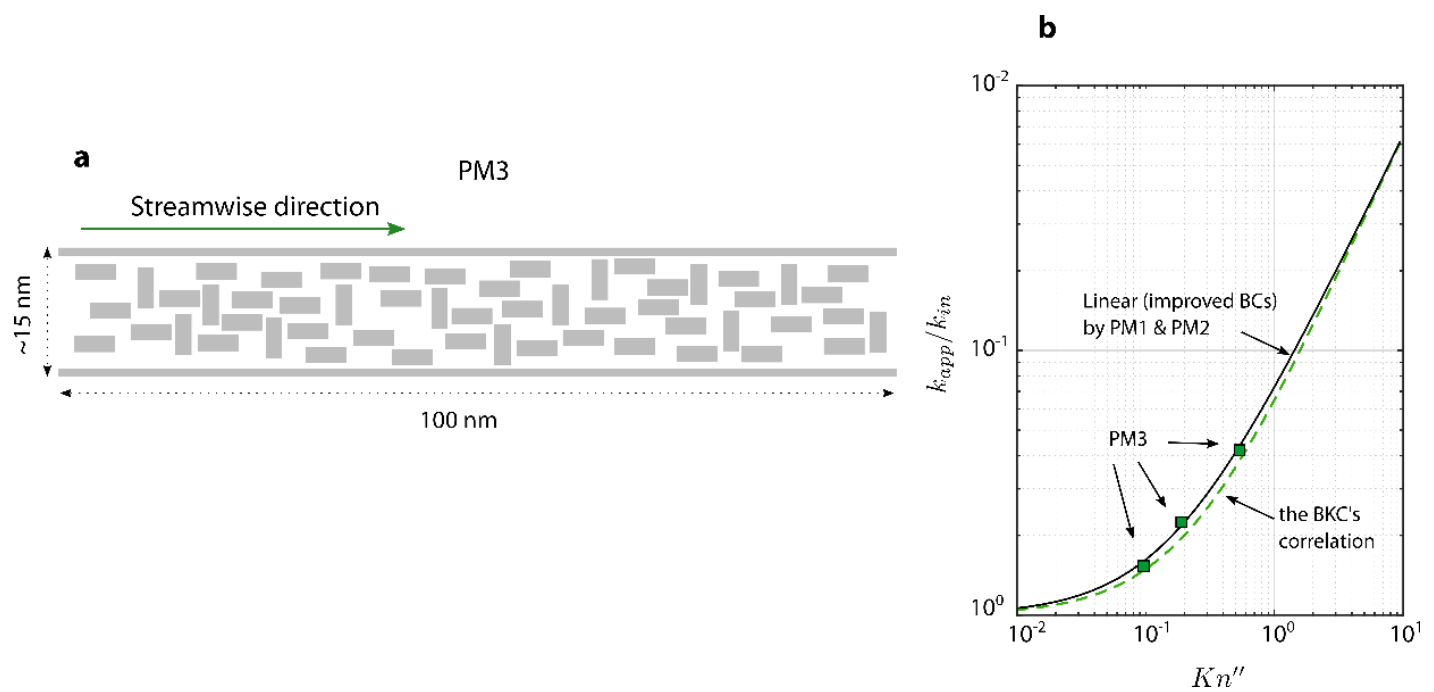

Fig. 6. Uniform solid slabs are randomly distributed within the PM3 in order to simulate complex pore structure pathways. Porosity is $\varepsilon=0.54$ and the intrinsic permeability is estimated by implementing the MBB BC as $k_{\text {in }}=4.50 \mathrm{e}-08\left(\mu \mathrm{m}^{2}\right)$ (converged at $\left.15000 \mathrm{ts}\right)$.

\subsection{Application of the Improved BC to Complex Porous Media}

Recent studies (Chen et al. 2015, Li et al. 2018, Zhao and Wang 2019) suggest that the reason for the overestimation of the Klinkenberg effect by using the $\mathrm{SBB}+\mathrm{SR} \mathrm{BC}$ is due to the complexity of pore structure. To investigate the impact of the structure of PM on the Klinkenberg effect, we applied the improved $\mathrm{BC}$ proposed here to a more complex pore 
network, which we identify as PM3 and whose structure is illustrated in Fig. 6a. Flowing pathways within PM3 are more tortuous than those within PM1 and PM2. The pore size follows a lognormal distribution where the mean is $3.7 \mathrm{~nm}$ and the logarithmic standard deviation is 0.384 . Gas atomic density and viscosity in the $3.8 \mathrm{~nm}$-sized pore (p5 in PM1) are used as the inputs for the flow simulation through PM3. The effective Knudsen number for PM3 is evaluated as in Equation (6).

In Fig. 6b, the estimated values of $k_{a p p} / k_{\text {in }}$ versus $K n^{\prime \prime}$ are evaluated at $K n^{\prime \prime}=$ 0.0967, 0.1894, and 0.5304, and the values fall onto the linear regression curve predicted for PM1 and PM2 and the BKC's correlation. We find that when the $K n^{\prime \prime}$ is evaluated, the

trend of $k_{a p p} / k_{\text {in }}$ does not depend on the configuration of individual pores but presents a universal fashion, a result which is also implied by the core-flooding experimental results obtained from different core samples shown in Fig. 5b and a DSMC study in Ref. (Yang and Weigand 2018). The good agreement between the estimated $k_{a p p} / k_{i n}$ and the BKC correlation shows a strong applicability the $\mathrm{MBB}+\mathrm{SR} \mathrm{BC}$ to the gas flow through complex pore structures.

\section{Discussion}

Because recent applications of LB simulations fail to reproduce the Klinkenberg effect when gas flows through micro-/nano-porous media, it is reasonable to question whether the mesoscale LB simulations can be improved to achieve numerical consistency. In this 
work, we leveraged atomistic non-equilibrium molecular dynamics (MD) simulation to improve the accuracy of simulating the finite-Kn flow by the LB method.

We found that MD simulations (Fig. 2) help identify the correct slip BCs in the LB simulation setup. We showed that when the improved BCs are implemented, the LB simulations of the gas transport and the Klinkenberg effect are quantitatively consistent with MD in terms of flow velocity and permeability. The improved BCs reasonably predict the dimensional velocity as in $\mathrm{MD}$; yet the classical $\mathrm{BCs}$ where the parallel velocities at the solid-gas interface are not properly addressed can lead to unphysical slip (Figs. 2a through 2d). Additionally, LB simulations with the improved BCs proposed here can quantitatively reproduce the permeability estimates from MD; while the LB simulations with the classical BCs (Figs. 4) overpredict the permeability. We also found the unphysical slip in the SBB BC cannot be numerically canceled out when one calculates the permeability correction factor, as the ratio of $k_{a p p}^{(S B B+S R)} / k_{i n}^{(S B B)}$ (Fig. 5).

Numerical comparisons of permeability correction factors from multi-scale source data (Fig. 5) testify the improvement achieved when implementing the improved BCs in LB simulations. The LB models implementing the classical BCs, both in this work and in the literature (Chen et al. 2015, Li et al. 2018, Prestininzi et al. 2016, Zhao and Wang 2019), significantly overestimate $k_{a p p} / k_{\text {in }}$ in the transitional flow region. When implementing the $\mathrm{MBB}+\mathrm{SR}$ and $\mathrm{MBB} \mathrm{BCs}, \mathrm{LB}$ models can fairly well reproduce $k_{a p p} / k_{\text {in }}$ values for ultra-tight rock, e.g., shale, and nanofluidic data for $0.01 \leq K n^{\prime} \leq 10$ (Fig. 5b). This 
shows the extended applicability of the improved LB method from continuum to transitional flow simulation.

Additionally, the LB simulations with improved BCs yield a general agreement with other classical non-LB approaches in estimating the permeability correction factor, especially the widely used BKC's correlation, which has been reflected in the results of PM1 and PM2 when $0.01 \leq K n^{\prime} \leq 10$ (Fig. 5b) and more complex PM, e.g., PM3, when $0.01 \leq K n^{\prime \prime} \leq 10$ (Fig. 6b).

Admittedly, alternative approaches have been proposed to improve the LB model for describing the finite- $K n$ flow including using multiple relaxation times (MRT) (Tao and Guo 2015, Wang et al. 2018), and high-order velocity sets (Kim et al. 2008). However, the approach proposed in this work has the distinct advantage to be relatively simple, which is coupled with the single relaxation time (SRT) and the widely implemented D2Q9 velocity set. The computational requirements of SRT are modest. Compared to MRT, SRT is much simpler in mathematical form and more computationally efficient. Studies showed that "a carefully optimized MRT will cost 10\%-20\% computational overhead than SRT model" (Succi and Succi 2018). Over the past decade, many debated whether MRT or SRT should be preferred. That MRT can outperform the SRT model in stability and accuracy is under the assumption that an optimum parameter set is found for conducting calculations within the MRT approach. However, as there is no universal guideline to find the optima, the superiority of MRT scheme cannot be taken for granted (Succi and Succi 2018). Lastly, studies show that high-order velocity set may not always be effective in improving the 
accuracy of LB method; the improved accuracy is not guaranteed by the increase of the velocity set order but depends on the flow type and the choice of the Gauss-Hermite quadrature (Kim et al. 2008, Meng and Zhang 2011). This, in turn, requires the prerequisite effort to testify the optimum order of the velocity set for a certain type of application.

\section{Conclusions}

In summary, the applicability of the LB method for slip and transitional flows in tight gas rocks, e.g. shale rocks, has been extended by addressing the slip boundary condition. Throughout this paper, the classical $\mathrm{BC}$ against an improved $\mathrm{BC}$ proposed here have been compared extensively in terms of dimensional flow velocity and permeability. Our results demonstrate that the improved BC is physically sound. Although widely applied throughout the past decade, classical BCs do not seem always suitable to simulate the finite- $K n$ gas flow, e.g., shale gas transport, within the LB method. This work also provides quantitative analysis concerning the overestimation of the Klinkenberg effect reported in previous studies for gas permeability, and it suggests that computational limitations inherent to the standard bounce-back (SBB) scheme may be the reason for the observed deviation compared to experiments. The results obtained when the improved $\mathrm{BC}$ is implemented are validated against multi-scale source data. The results suggest that the proposed mesoscopic approach is a computationally inexpensive and robust numerical tool to support core-flooding measurements and can unfold the consistent Klinkenberg effect observed from a multi-scale perspective. Because of its reliability, combined with modest 
computational cost, the method proposed here could be valuable for predicting the permeability of tight rock masses, including shale formations, which is essential for enhancing the accuracy of production models often used in the sector.

\section{Acknowledgments}

This is work is part of the Science for Clean Energy European (S4CE) research consortium funded by European Union's Horizon 2020 research and innovation program under grant agreement No.764810 (S4CE). The authors are grateful to Prof. John Shaw for stimulating discussions during his sabbatical at University College London, which was enabled, in part, by grant agreement number VP2-2017-023 from the Leverhulme Trust.

\section{References}

Ansumali, S. and I. V. Karlin (2002). Kinetic boundary conditions in the lattice boltzmann method. Physical Review E 66(2), 026311.

Apostolopoulou, M., R. Dusterhoft, R. Day, M. Stamatakis, M.-O. Coppens, and A. Striolo (2019). Estimating permeability in shales and other heterogeneous porous media: Deterministic vs. stochastic investigations. International Journal of Coal Geology 205, $140-154$.

Beskok, A. and G. E. Karniadakis (1999). Report: a model for flows in channels, pipes, and ducts at micro and nano scales. Microscale Thermophysical Engineering 3(1), 43-77.

Bhatia, S. K. and D. Nicholson (2006). Transport of simple fluids in nanopores: Theory and simulation. AIChE Journal 52(1), 29-38.

Bonnaud, P., Q. Ji, B. Coasne, R.-M. Pellenq, and K. Van Vliet (2012). Thermodynamics of water confined in porous calcium-silicate-hydrates. Langmuir 28(31), 11422-11432. 
Borner, A., F. Panerai, and N. N. Mansour (2017). High temperature permeability of fibrous materials using direct simulation Monte Carlo. International Journal of Heat and Mass Transfer 106, 1318-1326.

Bui, T., A. Phan, D. R. Cole, and A. Striolo (2017). Transport mechanism of guest methane in water-filled nanopores. The Journal of Physical Chemistry C 121(29), 15675-15686.

Cengel, Y. A. (2010). Fluid mechanics : fundamentals and applications / Yunus A. Äłengel, John M. Cimbala. (2nd ed. in SI units / adapted by Mehmet KanoÄŸlu. ed.). Boston, Mass.; London: McGraw-Hill Higher Education.

Chapman, S., T. G. Cowling, and D. Burnett (1990). The mathematical theory of nonuniform gases: an account of the kinetic theory of viscosity, thermal conduction and diffusion in gases. Cambridge university press.

Chen, L., L. Zhang, Q. Kang, H. S. Viswanathan, J. Yao, and W. Tao (2015). Nanoscale simulation of shale transport properties using the lattice Boltzmann method: permeability and diffusivity. Scientific reports 5, 8089.

Civan, F. (2010). Effective correlation of apparent gas permeability in tight porous media. Transport in porous media 82(2), 375-384.

Cornubert, R., D. d'Humières, and D. Levermore (1991). A Knudsen layer theory for lattice gases. Physica D: Nonlinear Phenomena 47(1-2), 241-259.

Dahm, K. and D. Visco (2014). Fundamentals of Chemical Engineering Thermodynamics. Nelson Education.

Darabi, H., A. Ettehad, F. Javadpour, and K. Sepehrnoori (2012). Gas flow in ultra-tight shale strata. Journal of Fluid Mechanics 710, 641-658.

Di Staso, G. (2018). Hybrid discretizations of the Boltzmann equation for the dilute gas flow regime. $\mathrm{PhD}$ dissertation, Technische Universiteit Eindhoven.

Fan, D. and A. Ettehadtavakkol (2017). Analytical model of gas transport in heterogeneous hydraulically-fractured organic-rich shale media. Fuel 207, 625-640.

Fan, X.-J., N. Phan-Thien, N. T. Yong, and X. Diao (2002). Molecular dynamics simulation of a liquid in a complex nano channel flow. Physics of Fluids 14(3), 1146-1153.

Fathi, E., I. Y. Akkutlu, et al. (2012). Lattice boltzmann method for simulation of shale gas transport in kerogen. Spe Journal 18(01), 27-37.

Gallivan, M. A., D. R. Noble, J. G. Georgiadis, and R. O. Buckius (1997). An evaluation of the bounce-back boundary condition for lattice Boltzmann simulations. International Journal for Numerical Methods in Fluids 25(3), 249-263. 
Ghanbarian, B. and F. Javadpour (2017). Upscaling pore pressure-dependent gas permeability in shales. Journal of Geophysical Research: Solid Earth 122(4), 2541-2552.

Guo, Z., B. Shi, T. Zhao, and C. Zheng (2007). Discrete effects on boundary conditions for the lattice Boltzmann equation in simulating microscale gas flows. Physical Review E 76(5), 056704.

Guo, Z. and C. Shu (2013). Lattice Boltzmann method and its applications in engineering, Volume 3. World Scientific.

Guo, Z., C. Zheng, and B. Shi (2008). Lattice Boltzmann equation with multiple effective relaxation times for gaseous microscale flow. Physical Review E 77(3), 036707.

Hadjiconstantinou, N. G. (2005). Validation of a second-order slip model for dilute gas flows. Microscale Thermophysical Engineering 9(2), 137-153.

Hadjiconstantinou, N. G. (2006). The limits of Navier-Stokes theory and kinetic extensions for describing small-scale gaseous hydrodynamics. Physics of Fluids 18(11), 111301.

He, X., Q. Zou, L. Luo, and M. Dembo (1997). Analytic solutions of simple flows and analysis of nonslip boundary conditions for the lattice Boltzmann BGK model. Journal of Statistical Physics 87(1-2), 115-136.

Horbach, J. and S. Succi (2006). Lattice Boltzmann versus molecular dynamics simulation of nanoscale hydrodynamic flows. Physical Review Letters 96(22), 224503.

Jasper, A. W. and J. A. Miller (2014). Lennard-Jones parameters for combustion and chemical kinetics modeling from full-dimensional intermolecular potentials. Combustion and Flame 161(1), 101-110.

Kalarakis, A., V. Michalis, E. Skouras, and V. Burganos (2012). Mesoscopic simulation of rarefied flow in narrow channels and porous media. Transport in porous media 94(1), 385398.

Kim, S. H., H. Pitsch, and I. D. Boyd (2008). Accuracy of higher-order lattice Boltzmann methods for microscale flows with finite Knudsen numbers. Journal of computational physics 227(19), 8655-8671.

Klinkenberg, L. (1941). The permeability of porous media to liquids and gases. In Drilling and production practice. American Petroleum Institute.

Krüger, T., H. Kusumaatmaja, A. Kuzmin, O. Shardt, G. Silva, and E. M. Viggen (2017). The lattice Boltzmann method. Springer International Publishing 10.

Landry, C. J., M. Prodanovic, and P. Eichhubl (2016). Direct simulation of supercritical gas flow in complex nanoporous media and prediction of apparent permeability. Ínternational Journal of Coal Geology 159, 120-134. 
Lasseux, D., F. J. V. Parada, and M. L. Porter (2016). An improved macroscale model for gas slip flow in porous media. Journal of Fluid Mechanics 805, 118-146.

Lemmon, E. W., M. O. McLinden, and D. G. Friend (2019). Thermophysical properties of fluid systems. In NIST Chemistry WebBook, NIST Standard Reference Database Number 69. Eds. P.J. Linstrom and W.G. Mallard, National Institute of Standards and Technology, Gaithersburg MD, 20899.

Li, X., J. Fan, H. Yu, Y. Zhu, and H. Wu (2018). Lattice Boltzmann method simulations about shale gas flow in contracting nano-channels. International Journal of Heat and Mass Transfer 122, 1210-1221.

Liu, C., G. Yin, and M. e. a. Li (2019). Shale permeability model considering bedding effect under true triaxial stress conditions. Journal of Natural Gas Science and Engineering, 102908.

Liu, J., Y. Qin, S. Zhang, and C. He (2019). Numerical solution for borehole methane flow in coal seam based on a new dual-porosity model. Journal of Natural Gas Science and Engineering, 102916.

Loyalka, S. and S. Hamoodi (1990). Poiseuille flow of a rarefied gas in a cylindrical tube: solution of linearized Boltzmann equation. Physics of Fluids A: Fluid Dynamics 2(11), 2061-2065.

Maurer, J., P. Tabeling, P. Joseph, and H. Willaime (2003). Second-order slip laws in microchannels for helium and nitrogen. Physics of Fluids 15(9), 2613-2621.

Meng, J., X. Gu, and D. R. Emerson (2018). Analysis of non-physical slip velocity in lattice Boltzmann simulations using the bounce-back scheme. Journal of computational science 28, 476-482.

Meng, J. and Y. Zhang (2011). Accuracy analysis of high-order lattice Boltzmann models for rarefied gas flows. Journal of Computational Physics 230(3), 835-849.

Moghaddam, R. N. and M. Jamiolahmady (2016). Slip flow in porous media. Fuel 173, 298-310.

Moghaddam, R. N. and M. Jamiolahmady (2017). Study of slip flow in unconventional shale rocks using lattice boltzmann method: Effects of boundary conditions and tmac. Transport in Porous Media 120(1), 115-139.

Niu, X.-D., S.-A. Hyodo, T. Munekata, and K. Suga (2007). Kinetic lattice Boltzmann method for microscale gas flows: issues on boundary condition, relaxation time, and regularization. Physical review E 76(3), 036711. 
Noble, D. R., S. Chen, J. G. Georgiadis, and R. O. Buckius (1995). A consistent hydrodynamic boundary condition for the lattice Boltzmann method. Physics of Fluids 7(1), 203-209.

Obliger, A., R. Pellenq, F.-J. Ulm, and B. Coasne (2016). Free volume theory of hydrocarbon mixture transport in nanoporous materials. The Journal of Physical Chemistry Letters 7(19), 3712-3717.

Phan, A., D. R. Cole, R. G. Weiß, J. Dzubiella, and A. Striolo (2016). Confined water determines transport properties of guest molecules in narrow pores. ACS nano 10(8), 76467656.

Phan, A. and A. Striolo (2019). Methane transport through hierarchical silica micromesoporous materials: From non-equilibrium atomistic simulations to phenomenological correlations. Microporous and Mesoporous Materials.

Prestininzi, P., A. Montessori, M. La Rocca, and S. Succi (2016). Reassessing the single relaxation time lattice Boltzmann method for the simulation of Darcy's flows. International Journal of Modern Physics C 27(04), 1650037.

Qomi, M. J. A., M. Bauchy, F.-J. Ulm, and R. J.-M. Pellenq (2014). Anomalous composition-dependent dynamics of nanoconfined water in the interlayer of disordered calcium-silicates. The Journal of chemical physics 140(5), 054515.

Rani, S., E. Padmanabhan, T. Bakshi, B. K. Prusty, and S. K. Pal (2019). Co2 sorption and rate characteristics in micropores of shales. Journal of Natural Gas Science and Engineering, 102903.

Ren, J., P. Guo, Z. Guo, and Z. Wang (2015). A lattice boltzmann model for simulating gas flow in kerogen pores. Transport in Porous Media 106(2), 285-301.

Song, W., J. Yao, Y. Li, H. Sun, and Y. Yang (2018). Fractal models for gas slippage factor in porous media considering second-order slip and surface adsorption. International Journal of Heat and Mass Transfer 118, 948-960.

Striolo, A. and D. R. Cole (2017). Understanding shale gas: Recent progress and remaining challenges. Energy \& Fuels 31(10), 10300-10310.

Succi, S. (2016). Lattice Boltzmann beyond Navier-Stokes: where do we stand? In AIP Conference Proceedings, Volume 1786, pp. 030001. AIP Publishing.

Succi, S., O. Filippova, H. Chen, and S. Orszag (2002). Towards a renormalized lattice boltzmann equation for fluid turbulence. Journal of Statistical physics 107(1-2), 261-278.

Succi, S. and S. Succi (2018). The Lattice Boltzmann Equation: For Complex States of Flowing Matter. Oxford University Press. 
Tang, G., W. Tao, and Y. He (2005a). Gas slippage effect on microscale porous flow using the lattice Boltzmann method. Physical Review E 72(5), 056301.

Tang, G., W. Tao, and Y. He (2005b). Lattice boltzmann method for gaseous microflows using kinetic theory boundary conditions. Physics of Fluids 17(5), 058101.

Tao, S. and Z. Guo (2015). Boundary condition for lattice Boltzmann modeling of microscale gas flows with curved walls in the slip regime. Physical Review E 91(4), 043305.

Toschi, F. and S. Succi (2005). Lattice Boltzmann method at finite Knudsen numbers. Europhysics Letters 69(4), 549.

Velasco, A., S. Friedman, M. Pevarnik, Z. Siwy, and P. Taborek (2012). Pressure-driven flow through a single nanopore. Physical Review E 86(2), 025302.

Verhaeghe, F., L.-S. Luo, and B. Blanpain (2009). Lattice Boltzmann modeling of microchannel flow in slip flow regime. Journal of Computational Physics 228(1), 147157.

Wang, J., L. Chen, Q. Kang, and S. S. Rahman (2016). The lattice boltzmann method for isothermal micro-gaseous flow and its application in shale gas flow: A review. International Journal of Heat and Mass Transfer 95, 94-108.

Wang, J., Q. Kang, Y. Wang, R. Pawar, and S. S. Rahman (2017). Simulation of gas flow in micro-porous media with the regularized lattice Boltzmann method. Fuel 205, 232-246.

Wang, Z., M. Wang, and S. Chen (2018). Coupling of high Knudsen number and non-ideal gas effects in microporous media. Journal of Fluid Mechanics 840, 56-73.

Wu, L., M. T. Ho, L. Germanou, X. Gu, C. Liu, K. Xu, and Y. Zhang (2017). On the apparent permeability of porous media in rarefied gas flows. Journal of Fluid Mechanics 822, 398-417.

$\mathrm{Xu}, \mathrm{S}$., Q. Feng, S. Wang, and Y. Li (2019). A 3d multi-mechanistic model for predicting shale gas permeability. Journal of Natural Gas Science and Engineering, 102913.

Yamaguchi, H., Y. Matsuda, and T. Niimi (2012). Tangential momentum accommodation coefficient measurements for various materials and gas species. In Journal of Physics: Conference Series, Volume 362, pp. 012035. IOP Publishing.

Yamaguchi, H., Y. Matsuda, and T. Niimi (2017). Molecular-dynamics study on characteristics of energy and tangential momentum accommodation coefficients. Physical Review E 96(1), 013116. 
Yang, G. and B. Weigand (2018). Investigation of the Klinkenberg effect in a micro/nanoporous medium by direct simulation Monte Carlo method. Physical Review Fluids 3(4), 044201.

Yu, H., J. Chen, Y. Zhu, F. Wang, and H. Wu (2017). Multiscale transport mechanism of shale gas in micro/nano-pores. International Journal of Heat and Mass Transfer 111, $1172-1180$.

Zhang, R., X. Shan, and H. Chen (2006). Efficient kinetic method for fluid simulation beyond the Navier-Stokes equation. Physical Review E 74(4), 046703.

Zhang, W., G. Meng, and X. Wei (2012). A review on slip models for gas microflows. Microfluidics and nanofluidics 13(6), 845-882.

Zhao, J., J. Yao, A. Li, M. Zhang, L. Zhang, Y. Yang, and H. Sun (2016). Simulation of microscale gas flow in heterogeneous porous media based on the lattice boltzmann method. Journal of Applied Physics 120(8), 084306.

Zhao, J., J. Yao, M. Zhang, L. Zhang, Y. Yang, H. Sun, S. An, and A. Li (2016). Study of gas flow characteristics in tight porous media with a microscale lattice boltzmann model. Scientific reports 6, 32393.

Zhao, T., H. Zhao, X. Li, Z. Ning, Q. Wang, W. Zhao, and J. Zhang (2018). Pore scale characteristics of gas flow in shale matrix determined by the regularized lattice Boltzmann method. Chemical Engineering Science 187, 245-255.

Zhao, Y. and Z. Wang (2019). Prediction of apparent permeability of porous media based on a modified lattice Boltzmann method. Journal of Petroleum Science and Engineering 174, 1261-1268.

Ziarani, A. S. and R. Aguilera (2012). Knudsen's permeability correction for tight porous media. Transport in porous media 91(1), 239-260. 